\title{
A INICIAÇÃO CIENTÍFICA NA GRADUAÇÃO EM ENFERMAGEM DA UNIVERSIDADE DE SÃO PAULO (1993 A 1996): ANÁLISE CRÍTICA
}

Ligia de Oliveira Pereira*

Adriana Inocenti**

Graciette Borges da Silva***

PEREIRA, L.de O.; INOCENTI, A.; SILVA, G.B.da. A iniciação científica na graduação em enfermagem da Universidade de São Paulo (1993 a 1996): análise crítica. Rev.latino-am.enfermagem, Ribeirão Preto, v. 7, n. 3 , p. 77-86, julho 1999.

Este estudo busca delinear o perfil da produção científica da graduação em enfermagem da Universidade de São Paulo de 1993 a 1996, identificando-lhe os traços principais e tendências. Para essa caracterização foram analisados os resumos dos artigos dos Simpósios de Iniciação Científica da USP (SICUSP) e dos Simpósios de Iniciação Científica da Escola de Enfermagem de Ribeirão Preto (SICEERP), através de uma classificação por tipos de pesquisa, temáticas e departamentos de origem. Observamos um predomínio de pesquisas de cunho descritivo, um número expressivo de trabalhos voltados para o estudo de aspectos sócio-culturais, dentre outros resultados.

UNITERMOS: pesquisa, estudantes de enfermagem

\section{INTRODUÇÃO}

Este estudo articula-se ao Projeto Integrado: "O processo de (re)produção do saber na pós-graduação em Enfermagem no Brasil", objetivo refletir sobre (e caracterizar) a produção do saber na enfermagem a partir do surgimento, na década de 70 , dos cursos de pós-graduação e de sua expansão posterior, que estão diretamente vinculados ao processo de modernização da universidade brasileira ${ }^{2}$.

Ele busca caracterizar a produção científica da graduação em enfermagem da Universidade de São Paulo nos anos de 1993 a 1996. Para enfocar este problema de investigação, pensamos ser importante traçar algumas considerações de ordem geral relativas à pesquisa em enfermagem no Brasil que, como em outras áreas, sofre mudanças articuladas ao contexto histórico do país. $\mathrm{O}$ primeiro trabalho relevante de pesquisa em enfermagem, de âmbito nacional, foi o Levantamento de Recursos e Necessidades de Enfermagem, realizado pela Associação Brasileira de Enfermagem entre 1956 e 1958, financiado pela Fundação Rockefeller, sendo que a primeira investigação documentada na área foi o "Censo de 1950". Nessa década, as temáticas mais investigadas relacionavam-se ao ensino de enfermagem e à profissão. Com a passagem da enfermagem para o $3^{\circ}$ grau, em 1961, a reforma universitária de 1968 e a criação dos cursos de pós-graduação "stricto sensu" na região sudeste, nos anos setenta, houve um grande avanço da pesquisa nessa área. Nessa década, os trabalhos publicados nos principais periódicos da citada área abordavam principalmente a metodologia de enfermagem e, nos anos oitenta, as pesquisas em enfermagem eram direcionadas para as necessidades do paciente, predominando, no conjunto dessa produção, no período de 1950 a 1980, as pesquisas descritivas e exploratórias, em detrimento de outras modalidades $^{3}$ (NOGUEIRA, 1982).

\footnotetext{
*Aluna do sexto semestre de graduação da Escola de Enfermagem de Ribeirão Preto da Universidade de São Paulo ** Aluna do oitavo semestre de graduação da Escola de Enfermagem de Ribeirão Preto da Universidade de São Paulo *** Professora Associada do Departamento de Enfermagem Psiquiátrica e Ciências Humanas da Escola de Enfermagem de Ribeirão Preto da Universidade de São Paulo, orientadora

${ }^{1}$ Esse projeto, coordenado por Silva, G.B, recebeu financiamento do CNPq para o período de agosto de 1995 a julho de 1997

${ }^{2}$ A reforma universitária, criada pela Lei 5.540/68, constituiu-se em uma reforma que buscava modernizar a universidade brasileira, ao lado de outras, de modernização do Estado. De cunho autoritário, essa reforma instituiu a generalização do vestibular unificado, a extinção da cátedra vitalícia, a criação de departamentos, semestralidade e divisão do currículo em dois ciclos (o básico e o profissionalizante). Buscou ainda a organização das instituições de ensino superior em universidades e a introdução efetiva dos cursos de pós-graduação "stricto sensu" (SILVA, 1994)

${ }^{3}$ Por exemplo a pesquisa experimental e a pesquisa qualitativa, segundo a classificação de CHIZOTTI (1995)
} 
Os Simpósios de Iniciação Científica da USP (SICUSP) surgiram em 1993 para divulgar as pesquisas financiadas pelo PIBIC (Programa Institucional de Bolsas de Iniciação Científica). Com a finalidade de dar oportunidade à divulgação de pesquisas de alunos vinculados a outros Programas ${ }^{4}$, apoiados por outros órgãos financiadores, foi criado, no mesmo ano, o Simpósio de Iniciação Científica da Escola de Enfermagem de Ribeirão Preto (SICEERP), que teve a duração de três anos (1993 a 1995).

Tendo em vista que, a partir de 1995, o SICUSP abriu espaço para a divulgação de trabalhos de Iniciação Científica de outras origens, não se restringindo mais aos do PIBIC, o referido Simpósio da Escola de Enfermagem de Ribeirão Preto perdeu a sua finalidade e, visando evitar a sobreposição de atividades, foi extinto em 1996.

A iniciação científica permite que o aluno de graduação tenha noções teóricas e metodológicas de pesquisa, buscando incentivar-lhe a capacidade de pensar e o espírito questionador. Esse contato com a pesquisa, além de enriquecer a formação escolar do aluno, fornecelhe o alicerce para a continuidade dos estudos nos programas de pós-graduação, principalmente se a opção feita for a carreira acadêmica.

No entanto, foi-nos possível observar que alguns bolsistas de iniciação científica estão insatisfeitos, devido à sobrecarga de tarefas que lhes são atribuídas, desvinculadas, muitas vezes, dos objetivos propostos pelos programas a que estão vinculados ${ }^{5}$.

As bolsas destinadas à iniciação científica surgiram em 1951 com a criação do Conselho Nacional de Pesquisa $(\mathrm{CNPq})$. Em suas origens, esse Conselho estava subordinado diretamente à Presidência da República e, em 1974, passou a denominar-se Conselho Nacional de Desenvolvimento Científico e Tecnológico, vinculando-se, então, à Secretaria de Planejamento da Presidência da República.

$\mathrm{O} \mathrm{CNPq}$ possui três superintendências ligadas às grandes áreas do saber, onze coordenadorias e setenta e um comitês assessores (CAs):

1-Superintendência das Engenharias, Ciências Exatas e da Terra, com três coordenadorias:-Ciências Exatas e da Terra, (6 CAs); Informática e Engenharia, (3 CAs); Engenharias II , (4 CAs);
2- Superintendência de Ciências da Vida, com cinco coordenadorias: -Zootecnia e Veterinária, (1 CA); Ciências Biológicas e Meio Ambiente, (2 CAs); Ciências Biomédicas, 2 (CAs); Ciências da Saúde, (3 CAs); Ciências Agronômicas, Florestais e de Alimentos, (3 CAs);

3- Superintendência de Ciências Humanas e Sociais, com três coordenadorias: -Ciências Humanas, (3 CAs); Ciências Sociais e Educação, (3 CAs); Ciências Sociais Aplicadas (2 CAs).

No $\mathrm{CNPq}$, a enfermagem está inserida na coordenadoria de Ciências da Saúde que possui 3 Comitês Assessores, entre os quais o de Saúde Complementar, que engloba as áreas de fonoaudiologia, fisioterapia, odontologia, educação física, enfermagem e terapia ocupacional. A partir de 1986, a inserção de uma representante da enfermagem no referido Comitê Assessor e de uma enfermeira como técnico de desenvolvimento científico do $\mathrm{CNPq}$, ambas atuando, pois, junto à Coordenadoria de Ciências da Saúde, representou a conquista de um espaço importante.

$\mathrm{O} \mathrm{CNPq}$ possui várias modalidades de bolsas sendo que atualmente, as de iniciação científica atingem mais de $40 \%$ dessas modalidades, incluindo, nesse percentual, as atribuídas pelo Programa Institucional de Bolsas de Iniciação Científica (PIBIC) e pelo Projeto Integrado (CNPq, 1996).

O PIBIC, criado em 1987, é um programa de abrangência nacional que incentiva as instituições universitárias a elaborarem políticas de pesquisa para a iniciação científica na graduação. Busca, principalmente, incentivar o aluno para as atividades de pesquisa, qualificando-o para os programas de pós-graduação, sendo a duração da bolsa de doze meses, podendo ser renovada até o término regular do curso de graduação (CNPq, 1996). As quotas/USP do PIBIC destinadas às várias unidades da USP aumentaram gradativamente de 1992 a 1996. Naquele ano, a Escola de Enfermagem de Ribeirão Preto recebeu cinco bolsas dessa modalidade, que passaram a sete em 1993, a treze, em 1994 e a dezesseis em 1995, número este que se manteve em 1996. As quotas para a Escola de Enfermagem de São Paulo, no citado período, foram sucessivamente quatro (1992), cinco (1993), seis (1994) e doze (1995 e 1996) ${ }^{6}$.

\footnotetext{
${ }^{4}$ A iniciação científica é contemplada por vários Programas, que explicitaremos no decorrer do texto: Programa Especial de Treinamento (PET/CAPES), Projeto Integrado de Pesquisa do CNPq e Programa Institucional de Bolsas de Iniciação Científica (PIBIC), entre outros

${ }^{5}$ Alguns alunos de iniciação científica costumam queixar-se de que estão sobrecarregados não só pelo fato de o curso de graduação em enfermagem ser integral, mas também por lhes ser solicitada a execução de tarefas alheias aos objetivos dos programas de iniciação científica, tais como: arrumação de armários, digitação de trabalhos desvinculados dos da bolsa, reuniões em horários destinados à graduação

${ }^{6}$ Segundo o coordenador do PIBIC na USP, a definição das quotas das várias unidades vinculada-se ao número de seus docentes com título de doutoramento relativamente ao total
} 
Quanto ao PET (Programa Especial de Treinamento) é financiado pela Coordenação de Aperfeiçoamento de Pessoal de Ensino Superior (CAPES), tendo sido criado em 1979. Hoje o número de seus bolsistas na USP é, aproximadamente, cento e setenta, sendo que a seleção ocorre anualmente.

Os objetivos do PET não estão centralizados somente na produção de um trabalho científico pelos bolsistas, mas procuram favorecer-lhes o desenvolvimento intelectual durante a formação acadêmica. $\mathrm{O}$ programa incentiva a participação em atividades extracurriculares incluindo eventos, cursos de língua estrangeira, discussões de temas variados, além de outras atividades sob a orientação de um tutor. $\mathrm{Na}$ Escola de Enfermagem de Ribeirão Preto esse programa foi criado em 1988, tendo no início quatro bolsistas e, atualmente, doze.

O programa referente a Projetos Integrados de

Pesquisa, ao qual esta pesquisa se vincula, incentiva a criação de projetos inter e multi-disciplinares, abrangendo a atuação de equipes no mesmo tema e a integração de grupos de pesquisadores.

Observa-se, dessa forma, que os órgãos financiadores da pesquisa (CNPq, CAPES, FAPESP) buscam incentivar, significativamente, o desenvolvimento e o aprimoramento do conhecimento científico no país, desde os cursos de graduação, visando, assim, a seus possíveis reflexos nos níveis pós-graduados, como o encurtamento do tempo de titulação.

\section{METODOLOGIA}

Como afirmamos anteriormente, o objetivo principal deste estudo é apreender as características básicas das pesquisas de iniciação científica, realizadas nas Escolas de Enfermagem de Ribeirão Preto e de São Paulo da Universidade de São Paulo, no período de 1993 a 1996.

Seus pressupostos gerais são que as pesquisas de enfermagem produzidas na Iniciação Científica, como em outros níveis de ensino, tendem a refletir as características dessa área em seu conjunto, e que tais características são historicamente determinadas (ou condicionadas). Mais especificamente, considerando que, de maneira geral, os orientadores da graduação e da pósgraduação tendem a ser os mesmos, supomos que as temáticas abordadas bem como a metodologia de investigação de ambos os níveis tenderão a apresentar convergências expressivas.

A trajetória percorrida para a elaboração do presente trabalho pode ser didaticamente dividida em dois momentos. Em uma fase preliminar foram realizadas leituras sobre metodologia científica, documentos referentes às agências de fomento à pesquisa e trabalhos relativos à pesquisa em enfermagem. Posteriormente foram analisados todos os resumos referentes à área de enfermagem, contidos nos Anais de Iniciação Científica da USP (SICUSP) e nos Anais de Iniciação Científica da Escola de Enfermagem de Ribeirão Preto ( SICEERP ) no período referido. Os citados Anais constituíram a matriz dos dados da produção que analisamos, a partir de uma classificação por: temáticas, tipos de pesquisa (experimental, analítica e descritiva) e procedência (departamentos da EE e da EERP-USP). Vale ressaltar que os departamentos da Escola de Enfermagem de São Paulo são: Enfermagem Médico-Cirurgica (ENC), Orientação Profisional (ENO), Enfermagem Materno Infantil e Psiquiátrica (ENP) e os departamentos da Escola de Enfermagem de Ribeirão Preto são: Enfermagem Geral e Especializada (EGE), Enfermagem Psiquiátrica e Ciências Humanas (EPCH), Enfermagem MaternoInfantil e Saúde Publica (MISP).

Visando ainda complementar este estudo, realizamos entrevistas com docentes (orientadores de bolsistas de iniciação científica e tutora do PET) e com a secretária da Comissão de Pesquisa da EERP-USP ${ }^{7}$.

Através dos itens classificatórios já citados, buscamos delinear o perfil dessa produção, identificandolhe os traços principais e tendências, em uma análise comparativa, que contemplasse simultaneamente seus aspectos internos e externos, visualizados como interdependentes e historicamente situados.

\section{APRESENTAÇÃO E ANÁLISE DOS DADOS}

Para o estudo feito, foram levantados e analisados os temas e respectivos conteúdos de todos os resumos referentes à área de enfermagem publicados, como já mencionamos, no SICUSP e no SICEERP no período de 1993 a 1996, constituindo um total de 249 trabalhos (139 e 110, respectivamente). Uma primeira leitura possibilitou a identificação dos temas recorrentes e dos conteúdos análogos ou próximos abordados, da qual emergiu uma proposta de classificação que foi discutida e testada no grupo de pesquisa durante todo o processo de análise. Essa classificação abrange os seguintes aspectos

\footnotetext{
${ }^{7}$ A finalidade dessas entrevistas foi obter informações a respeito das principais agências de fomento e subsídios para o entendimento da pesquisa em enfermagem e, mais especificamente, sobre a pesquisa na graduação
} 
(ordenados em ordem alfabética) ensino; estados/ variáveis/categorias biológicos, químicos, físicos; estados/variáveis/categorias psíquicos, comportamentais; estados/variáveis/categorias sócio-culturais; revisão de literatura; serviços de saúde; trabalho.

Para a classificação feita, decidimos não nos ater apenas aos títulos dos trabalhos, porque pudemos identificar, em alguns casos, que aqueles se distanciavam dos conteúdos dos resumos.

Da categoria ensino, constaram resumos sobre questões programáticas de ensino formal/regular e de cursos extracurriculares, programas educativos e de orientação em saúde. Como exemplos, temos: "O ensino de comunicação em enfermagem - um desafio"(SICUSP, 1995, p.46); "O trabalho extracurricular do aluno de graduação em enfermagem - relato de experiência na área de Saúde Ambiental" (SICEERP, 1993, p.45); "Educação para a saúde: AIDS em instituições escolares" (SICEERP, 1994, p.33); "Orientação postural aos escolares em escolas da rede pública de ensino de $1^{\circ}$ e $2^{\circ}$ grau" (SICUSP, 1995, p.41).

Em estados/variáveis/categorias biológicos, químicos, físicos foram incluídos os resumos que tratavam de aspectos ligados ao funcionamento orgânico, a drogas e a aspectos nutricionais. Podemos citar: "Estudo dos valores de pressão arterial obtidos de indivíduos com hipertensão leve em diferentes posições" (SICUSP, 1995, p.42); "Efeitos da microinjeção intramigdalóide de diazepam sobre o comportamento de ratos no labirinto em cruz elevado"(SICEERP, 1994, p. 10); "Caracterização da criança de risco nutricional através da avaliação do Gráfico para Acompanhamento do Crescimento em uma Unidade Básica de Saúde de Ribeirão Preto-SP" (SICEERP, 1993, p. 33).

$\mathrm{O}$ item estados/variáveis/categorias psíquicos e comportamentais englobou assuntos referentes a comportamentos e sentimentos das pessoas frente a situações específicas e medidas de percepção como, por exemplo, "Mensuração da percepção social do enfermeiro: um enfoque da Psicofísica" (SICEERP, 1993, p. 28); "Níveis de ansiedade entre alunos de graduação em enfermagem"(SICUSP, 1995, p.42).

O tópico sobre estados/variáveis/categorias sócio-culturais abrange trabalhos que abordam conhecimentos, informações, valores, práticas de saúde em camadas sociais específicas. São exemplos deste item: "Conhecimento entre escolares sobre o funcionamento do sistema reprodutor masculino e feminino: necessidade de orientação" (SICEERP, 1993, p. 38); "Estudo da forma de expressão da mulher sobre sua menstruação" (SICUSP, 1993, p. 14); "Repercussão de atividade de promoção de saúde e qualidade de vida em uma população adulta idosa em um bairro de Ribeirão Preto"(SICUSP, 1995, p. 51).

$\mathrm{O}$ tema revisão de literatura incluiu pesquisas direcionadas a levantamentos bibliográficos de áreas específicas do saber e a respectiva análise. Temos como exemplos, entre outros: "Resíduos perigosos - pesquisa bibliográfica de 1989 a 1993" (SICEERP, 1994, p. 16); "Análise das publicações sobre câncer em enfermagem" (SICEERP, 1993, p. 24).

Quanto ao tópico serviços de saúde, refere-se a pesquisas voltadas para o estudo da problemática geral da assistência à saúde ou para questões a ela relacionadas, focalizando instituições concretas. Os resumos referidos intitulam-se: "Buscando a eqüidade dos serviços de saúde - o papel da gerência nas Unidades Básicas de Saúde no município de Ribeirão Preto"(SICEERP, 1995, p.21); "Caracterização das Unidades de Terapia Intensiva do Município de São Paulo"(SICUSP, 1995, p.57). Surpreendentemente, apenas dois trabalhos foram classificados nessa categoria, evidenciando que uma das discussões centrais no preparo do profissional de enfermagem não está sendo pesquisada pelos alunos de iniciação científica.

Finalmente o item trabalho engloba assuntos sobre a problemática geral ou específica das características de atividades laborais das categorias que compõem a enfermagem, relativas à saúde/doença de seus trabalhadores ou à saúde/doença de outras categorias profissionais. Podem ser citados como exemplos: "Supervisão: a perspectiva dos enfermeiros em serviços hospitalares"(SICEERP, 1995, p. 18); “Assistência à criança: uma visão panorâmica do trabalho de enfermagem em uma Unidade Básica de Saúde" (SICUSP, 1995, p. 47); "Trabalhadores metalúrgicos: acidentes de trabalho ocorridos em empresas de Ribeirão Preto (SP)" (SICEERP, 1994, p. 36).

É necessário esclarecer que encontramos dificuldade em enquadrar alguns resumos na classificação adotada, pelo fato de estes evidenciarem conteúdos de zonas limítrofes do conhecimento ou passíveis de serem classificados em mais de um tópico. Entretanto, a decisão final buscou priorizar o objetivo central contido, implícita ou explicitamente, no resumo apresentado. Ilustrando: se a questão em estudo fosse o câncer de mama em uma determinada população, buscando descrever os aspectos relativos ao diagnóstico, tratamento ou a sua fisiopatologia, esse trabalho seria enquadrado no tópico estados/variáveis/categorias biológicos. No entanto, quando a questão central era apreender o conhecimento dos profissionais de enfermagem sobre o câncer de mama, esse tipo de pesquisa era enquadrado no item estados/ variáveis/categorias sócio-culturais. Buscaremos, neste momento, apresentar e analisar os dados referentes às temáticas abordadas, correspondentes ao período em estudo. Conforme pode ser visualizado nas Tabelas $1 \mathrm{e}$ 2 , encontramos um maior número de publicações referentes aos aspectos estados/variáveis/categorias 
sócio-culturais em todos os anos em análise, nos Simpósios de Iniciação Científica da USP e da Escola de Enfermagem de Ribeirão Preto (respectivamente, 59,7\% e $49 \%$ dos totais). Constatamos, portanto, que a problemática abarcada por esse item constitui-se na problemática central das pesquisas na graduação.

Tabela 1 - Classificação dos resumos da área de enfermagem dos anais dos Simpósios de Iniciação Científica da Universidade de São Paulo (SICUSP) no período de 1993-1996, segundo sua temática

\begin{tabular}{|c|c|c|c|c|c|c|c|c|c|}
\hline \multirow{3}{*}{ Temática } & \multicolumn{2}{|c|}{1993} & \multicolumn{2}{|c|}{1994} & \multicolumn{2}{|c|}{1995} & \multicolumn{2}{|c|}{1996} & \multirow{3}{*}{$\begin{array}{c}\text { TOTAL } \\
\mathrm{N}^{\circ}\end{array}$} \\
\hline & $\mathrm{N}^{\circ}$ & $\%$ & $\mathrm{~N}^{\circ}$ & $\%$ & $\mathrm{~N}^{\circ}$ & $\%$ & $\mathrm{~N}^{\circ}$ & $\%$ & \\
\hline & & & & & & & & & \\
\hline $\begin{array}{l}\text { AspectosBiológicos, Químicos, } \\
\text { Físicos }\end{array}$ & 2 & 22,2 & 7 & 36,8 & 8 & 17,4 & 11 & 16,9 & 28 \\
\hline Aspectos Sócio-Culturais & 5 & 55,6 & 9 & 47,4 & 28 & 60,9 & 41 & 63,1 & 83 \\
\hline Ensino & - & - & - & - & 3 & 6,5 & 2 & 3,1 & 5 \\
\hline Trabalho & 1 & 11,1 & - & - & 2 & 4,3 & 3 & 4,6 & 6 \\
\hline Serviços de Saúde & - & - & - & - & 1 & 2,2 & - & - & 1 \\
\hline Revisão de Literatura & - & - & 2 & 10,5 & 2 & 4,3 & 8 & 12,3 & 12 \\
\hline AspectosPsíquicos & 1 & 11,1 & 1 & 5,3 & 2 & 4,3 & - & - & 4 \\
\hline TOTAL & 9 & 100 & 19 & 100 & 46 & 100 & 65 & 100 & 139 \\
\hline
\end{tabular}

Tabela 2 - Classificação dos resumos dos Simpósios dos anais de Iniciação Científica da Escola de Enfermagem de Ribeirão Preto (SICEERP) no período de 1993-1995, segundo sua temática

\begin{tabular}{lccccccc} 
& \multicolumn{2}{c}{1993} & \multicolumn{2}{c}{1994} & \multicolumn{2}{c}{ TOTAL } \\
Temática & $\mathrm{N}^{\circ}$ & $\%$ & $\mathrm{~N}^{\circ}$ & $\%$ & $\mathrm{~N}^{\circ}$ & $\%$ & $\mathrm{~N}^{\circ}$ \\
\hline Aspectos Biológicos, Qúrmicos, Físicos & 7 & 20,0 & 7 & 18,4 & 7 & 18,9 & 21 \\
Aspectos Sócio-Culturais & 17 & 48,6 & 17 & 44,7 & 20 & 54,1 & 54 \\
Ensino & 1 & 2,9 & 3 & 7,9 & 2 & 5,4 & 6 \\
Trabalho & 1 & 2,9 & 4 & 10,5 & 2 & 5,4 & 7 \\
Serviços de Saúde & - & - & - & - & 1 & 2,7 & 1 \\
Revisão de Literatura & 7 & 20,0 & 4 & 10,5 & 3 & 8,1 & 14 \\
AspectosPsíquicos & 2 & 5,7 & 3 & 7,9 & 2 & 5,4 & 7 \\
\hline TOTAL & 35 & 100 & 38 & 100 & 37 & 100 & 110 \\
\hline
\end{tabular}

Por que isso ocorre?. De um lado, a explicação pode estar no maior peso relativo das ciências sociais no elenco das disciplinas que compõem os programas atualmente vigentes na pós-graduação dos cursos aqui enfocados ou nos conteúdos programáticos das disciplinas que tratam direta ou indiretamente de questões metodológicas. De outro, releva-se a necessidade das docentes-enfermeiras de adquirir conhecimento nessa área (e de transmiti-lo a seus orientandos da graduação e da pós-graduação), em consonância com a ideologia 
humanista e holística, tão presente na enfermagem, de valorização dos aspectos sócio-culturais e psíquicos do ser humano, aspectos estes que têm um espaço restrito nos currículos de graduação. A orientação de alunos de iniciação científica da USP usualmente está a cargo de professores com titulo de doutoramento (ou acima), egressos, em sua grande maioria, dos Programas de PósGraduação da área de enfermagem da própria USP. Uma análise do elenco de disciplinas do Doutorado interunidades $^{8}$, especificamente credenciadas para esse Programa, num total de catorze, mostra que 50\% delas são de cunho epistemológico e teórico-metodológico, $43 \%$, de cunho psico-social ou preocupadas com a assistência e 7\%, de cunho técnico-biológico.

ANGERAMI (1991), ao analisar as 18 teses produzidas no referido Programa de Doutoramento quanto aos marcos teóricos utilizados e sua origem, decorridos oito anos de sua criação, constatou que 17,85\% delas baseavam-se nas teorias de enfermagem; 3,57\% associavam essa teoria a outras e, quanto às demais, $17,85 \%$ estavam embasadas nas Ciências Biológicas; 42,85\%, nas Ciências Humanas; 10,71\%, nas Ciências Econômicas e Administrativas e 7,11\%, na Ciência Médica, dados indicativos, segundo essa autora, "do predomínio das Ciências Humanas e Sociais sobre as demais como fonte do referencial teórico utilizado pelos autores"(p.16), tendência que se mantém atualmente.

Por sua vez, o ciclo básico do curso de graduação da EERP-USP, com 1.170 horas, enfatiza as disciplinas de conteúdo biológico, que correspondem a 780 horas desse total, portanto, a $66,7 \%$, comparativamente às de ciências humanas, que correspondem a $25,6 \%$ (300 horas), sendo que 90 horas $(7,7 \%)$ das 1.170 citadas referem-se à estatística $(60$ horas) e a práticas esportivas (30 horas).

$\mathrm{O}$ item estados/variáveis/categorias biológicos, químicos, físicos, apesar de ocupar o segundo lugar na tipologia que construímos, o número de trabalhos por ele englobados é reduzido frente ao anteriormente citado. Pode-se dizer ainda que 33,3\% dos trabalhos apresentados no SICEERP, enquadrados nesse item, estiveram sob orientação de docentes não pertencentes à Escola de Enfermagem ${ }^{9}$. No que se refere aos tópicos ensino, trabalho e estados/variáveis/categorias psíquicos, observamos um número semelhante de trabalhos incluídos nessas temáticas no período em estudo. Vale ainda ressaltar algumas considerações de ordem geral. Observamos, dentro da produção analisada, que um trabalho foi apresentado em dois anos consecutivos com diferentes títulos, porém com o seu conteúdo praticamente inalterado. Detectamos, ainda, que alguns resumos não explicitaram a população em estudo, outros, não continham a metodologia adotada, registrando-se também a presença de trabalhos de conteúdo ambíguo sem explicação de seus objetivos. $\mathrm{Na}$ análise dos dados, evidenciou-se a presença de vários resumos cujo objetivo era realizar um mero levantamento de opiniões; outros, limitaram-se a descrever os tipos de respostas mais freqüentes a um dado problema, sem nenhum aprofundamento teórico-metodológico. THIOLLENT (1982), critica as pesquisas de opinião, que não considera científicas, afirmando que: "Um dos efeitos mais perniciosos da pesquisa de opinião pública consiste precisamente em ordenar que as pessoas respondam a perguntas que não se colocam ou, ainda, que respondam a uma pergunta diferente da pergunta colocada, sendo que a interpretação apenas registra o mal-entendido" (p.141).

Nas Tabelas 3 e 4 identificamos um maior número de trabalhos produzidos pelo Departamento de Enfermagem Geral e Especializada (EGE) em todos os anos deste estudo, o que pode-se explicar, em parte, pelo fato de esse departamento possuir um número maior de docentes ou mais docentes vinculados a programas de Iniciação Científica ${ }^{10}$.

\footnotetext{
${ }^{8}$ Esse Programa é o mais antigo da enfermagem e conjuga disciplinas das Escolas de Enfermagem de Ribeirão Preto e de São Paulo, além das mencionadas

${ }^{9}$ No ano de 1994, houve um trabalho que esteve sob a orientação de professores do Departamento de Psicobiologia da Faculdade de Filosofia Ciências e Letras de Ribeirão Preto da Universidade de São Paulo e um trabalho sob orientação de um professor do Departamento de Oftalmologia e Otorrinolaringologia da Faculdade de Medicina de Ribeirão Preto da Universidade de São Paulo. Em 1995, houve apresentação de um trabalho sob orientação de docentes do Departamento de Parasitologia, Imunologia e Microbiologia, um trabalho sob orientação do Departamento de Oftalmologia e Otorrinolaringologia, ambos da Faculdade de Medicina de Ribeirão Preto da Universidade de São Paulo e um trabalho sob orientação do Departamento de Psicobiologia da Faculdade de Filosofia, Ciências e Letras de Ribeirão Preto da Universidade de São Paulo

${ }^{10}$ A Escola de Enfermagem de Ribeirão Preto da Universidade de São Paulo possui, hoje, um total de sessenta e quatro docentes distribuídos em três departamentos: Enfermagem Geral e Especializada com trinta docentes; Enfermagem Materno-Infantil e Saúde Pública com vinte e três docentes e Enfermagem Psiquiátrica e Ciências Humanas com 11 docentes. A Escola de Enfermagem da Universidade de São Paulo possui, hoje, um total de setenta e oito docentes distribuídos em quatro departamentos: Enfermagem Médico-Cirúrgica com vinte e dois docentes, Enfermagem Materno-Infantil e Psiquiátrica com vinte e cinco docentes, Enfermagem de Saúde Coletiva com dezessete docentes e Departamento de Orientação Profissional com catorze docentes.(USP, 1996)
} 
Tabela 3 - Distribuição do total de pesquisas apresentadas nos anais dos Simpósios de Inciação Científica da Universidade de São Paulo no período de 1993-1996, segundo os departamentos da Escola de Enfermagem e da Escola de Enfermagem de Ribeirão Preto

\begin{tabular}{|c|c|c|c|c|c|c|c|c|c|c|}
\hline \multirow{3}{*}{\multicolumn{2}{|c|}{ Departamento }} & \multicolumn{2}{|c|}{1993} & \multicolumn{2}{|c|}{1994} & \multicolumn{2}{|c|}{1995} & \multicolumn{2}{|c|}{1996} & \multirow{3}{*}{$\begin{array}{c}\text { TOTAL } \\
\mathrm{N}^{\circ}\end{array}$} \\
\hline & & $\mathrm{N}^{\circ}$ & $\%$ & $\mathrm{~N}^{\circ}$ & $\%$ & $\mathrm{~N}^{\circ}$ & $\%$ & $\mathrm{~N}^{\circ}$ & $\%$ & \\
\hline & & & & & & & & & & \\
\hline MISP & & 1 & 11,1 & 5 & 26,3 & 16 & 34,8 & 21 & 32,3 & 43 \\
\hline $\mathrm{EGE}$ & & 3 & 33,3 & 6 & 31,6 & 14 & 30,4 & 23 & 35,4 & 46 \\
\hline $\mathrm{EPCH}$ & & 1 & 11,1 & 2 & 10,5 & 2 & 4,3 & 2 & 3,1 & 7 \\
\hline $\mathrm{ENC}$ & & 1 & 11,1 & 1 & 5,3 & 7 & 15,2 & 7 & 10,8 & 16 \\
\hline ENS & & 3 & 33,3 & 4 & 21,1 & 5 & 10,9 & 7 & 10,8 & 19 \\
\hline ENO & & 0 & 0,0 & 0 & 0,0 & 1 & 2,2 & 0 & 0,0 & 1 \\
\hline ENP & & 0 & 0,0 & 1 & 5,3 & 1 & 2,2 & 4 & 6,2 & 6 \\
\hline Outros* & & 0 & 0,0 & 0 & 0,0 & 0 & 0,0 & 1 & 1,5 & 1 \\
\hline TOTAL & & 9 & 100 & 19 & 100 & 46 & 100 & 65 & 100 & 139 \\
\hline
\end{tabular}

* Esse item refere-se a um trabalho que não explicita o orientador

Tabela 4 - Distribuição do total de pesquisas apresentadas nos anais dos Simpósios de Iniciação Científica da Escola de Enfermagem de Ribeirão Preto, no período de 1993-1995, segundo os departamentos dessa escola

\begin{tabular}{lccccccc} 
& \multicolumn{2}{c}{ Ano } & \multicolumn{2}{c}{1994} & \multicolumn{2}{c}{1995} & \multicolumn{2}{c}{ TOTAL } \\
Departamento & $\mathrm{N}^{\circ}$ & $\%$ & $\mathrm{~N}^{\circ}$ & $\%$ & $\mathrm{~N}^{\circ}$ & $\%$ & $\mathrm{~N}^{\circ}$ \\
\hline MISP & 17 & 48,6 & 9 & 23,7 & 13 & 35,1 & 39 \\
EGE & 13 & 37,1 & 17 & 44,7 & 18 & 48,6 & 48 \\
EPCH & 3 & 8,6 & 10 & 26,3 & 2 & 5,4 & 15 \\
Orientador não especificado & - & - & - & - & 1 & 2,7 & 1 \\
Outros* & 2 & 5,7 & 2 & 5,3 & 3 & 8,1 & 7 \\
\hline TOTAL & 35 & 100 & 38 & 100 & 37 & 100 & 110 \\
\hline
\end{tabular}

* Departamentos não pertencentes à Escola de Enfermagem de Ribeirão Preto

No que se refere à Escola de Enfermagem-USP, a relação tamanho do departamento e volume da produção estudada nem sempre ocorreu, pois o departamento que apresentou maior produção, o de Enfermagem de Saúde Coletiva (ENS), é um dos menores, tendo apresentado, no SICUSP, um total de 19 trabalhos (ver Tabela 3).

$\mathrm{Na}$ Tabela 4 pode-se observar que $30,2 \%$ das pesquisas apresentadas no SICUSP foram produzidas pela EE-USP e $69,8 \%$ pela EERP-USP. Essa tabela mostra ainda que, no conjunto, o número de trabalhos apresentados pela Escola de Enfermagem de Ribeirão Preto é mais que o dobro dos trabalhos apresentados pela Escola de Enfermagem de São Paulo, o que supomos se explique pelo menor número de docentes ligados a programas de Iniciação Científica nesta última.

Para a análise dos resumos quanto aos tipos de pesquisa, adotamos a tipologia de KERLINGER (1964) que as classifica em descritiva, analítica e experimental. Entretanto, tivemos dificuldade em enquadrar alguns 
trabalhos $(17,7 \%)$ devido ao fato de apresentarem, em seus resumos, pouca ou nenhuma informação de cunho teórico-metodológico, tendo optado por enquadrá-los no tópico "sem especificação"(Tabelas 4 e 5).

Nas Tabelas 5 e 6 observa-se um maior número de trabalhos classificados como pesquisa descritiva.

Tabela 5 - Classificação dos resumos dos anais dos Simpósios de Iniciação Científica da Escola de Enfermagem de Ribeirão Preto (SICEERP) segundo os tipos de pesquisa

\begin{tabular}{|c|c|c|c|c|c|c|c|c|}
\hline \multirow{3}{*}{\multicolumn{2}{|c|}{ Tipos de pesquisa }} & \multicolumn{2}{|c|}{1993} & \multicolumn{2}{|c|}{1994} & \multicolumn{2}{|c|}{1995} & \multirow{3}{*}{$\begin{array}{c}\text { TOTAL } \\
\mathrm{N}^{\circ}\end{array}$} \\
\hline & & $\mathrm{N}^{\circ}$ & $\%$ & $\mathrm{~N}^{\circ}$ & $\%$ & $\mathrm{~N}^{\circ}$ & $\%$ & \\
\hline & & & & & & & & \\
\hline Analitica & & 2 & 5,7 & 4 & 10,5 & 5 & 13,5 & 11 \\
\hline Descritiva & & 29 & 82,9 & 19 & 50,0 & 19 & 51,4 & 67 \\
\hline Experimental & & 2 & 5,7 & 2 & 5,3 & 4 & 10,8 & 8 \\
\hline Sem especificação & & 2 & 5,7 & 13 & 34,2 & 9 & 24,3 & 24 \\
\hline TOTAL & & 35 & 100 & 38 & 100 & 37 & 100 & 110 \\
\hline
\end{tabular}

Tabela 6 - Classificação dos resumos dos anais dos Simpósios de Iniciação Científica da USP (SICUSP) no período de 1993-1996, segundo os tipos de pesquisa

\begin{tabular}{lccccccccc} 
& & \multicolumn{2}{c}{1994} & \multicolumn{2}{c}{1995} & & 1996 & \multicolumn{2}{c}{ TOTAL } \\
Tipos de pesquisa & $\mathrm{N}^{\circ}$ & $\%$ & $\mathrm{~N}^{\circ}$ & $\%$ & $\mathrm{~N}^{\circ}$ & $\%$ & $\mathrm{~N}^{\circ}$ & $\%$ & $\mathrm{~N}^{\circ}$ \\
\hline Analitica & - & - & - & - & 4 & 8,7 & 12 & 18,4 & 16 \\
Descritiva & 8 & 88,9 & 14 & 73,7 & 33 & 71,7 & 46 & 70,8 & 98 \\
Experimental & 1 & 11,1 & 1 & 5,3 & 2 & 4,3 & 0 & 0,0 & 5 \\
Sem especificação & - & - & 4 & 21,0 & 7 & 15,2 & 7 & 10,8 & 20 \\
\hline TOTAL & 9 & 100 & 19 & 100 & 46 & 100 & 65 & 100 & 139 \\
\hline
\end{tabular}

Esse tipo de pesquisa, caracteriza-se pela ausência de hipóteses, estando usualmente ligado ao estudo de fenômenos pouco conhecidos (e pouco investigados), sendo que a descrição constitui uma fase preliminar fundamental das pesquisas que pretendem interpretar (explicar) os fatos que estudam. Entretanto, se não for este o caso, o predomínio expressivo desse tipo de pesquisa em dada área pode significar um relativo despreparo de seus profissionais na realização de investigações científicas e, portanto, impossibilidade de aprofundamento metodológico e de interpretações que signifiquem avanços do conhecimento (o que raramente acontece com a pesquisa descritiva).

A análise dos resumos revelou também que existe uma produção reduzida de pesquisas experimentais na enfermagem e que estas foram orientadas por docentes de outras áreas do saber.

\section{CONSIDERAÇÕES FINAIS}

A análise da produção científica da graduação em enfermagem da Universidade de São Paulo, nos anos de 1993 a 1996, publicada nos Anais dos Simpósios de Iniciação Científica da USP (SICUSP, 1993, 1996) e da Escola de Enfermagem de Ribeirão Preto-USP (SICEERP), foi o objeto desta pesquisa, alicerçada na abordagem histórico-estrutural. Nela, identificamos as seguintes características principais: um número expressivo de trabalhos voltados para o estudo de aspectos sócio-culturais, em todo o período enfocado $(59,7 \%$ e $45 \%$ dos totais de resumos da área de enfermagem publicados no SICUSP e SICEERP, respectivamente), constituindo-se na temática central das pesquisas da graduação; um índice elevado de pesquisas de cunho descritivo, relativamente às analíticas e experimentais (segundo a tipologia de Kerlinger); a produção 
relativamente elevada oriunda do Departamento de Enfermagem Geral e Especializada (EGE) da Escola de Enfermagem de Ribeirão Preto-USP (o maior departamento dessa unidade) e a produção maior de trabalhos produzidos por essa Escola em comparação com a Escola de Enfermagem de São Paulo.

A primeira característica apontada pode-se explicar pela existência na pós-graduação de um grande número de disciplinas oriundas das ciências sociais, refletindo na produção científica da graduação, uma vez que os orientadores geralmente são os mesmos, ou surge da necessidade em adquirir conhecimentos dessa área e transmiti-los a seus orientandos, visto que há um predomínio das ciências biológicas nos currículos de graduação. A segunda afirmação pode estar relacionada ao relativo despreparo dos profissionais da enfermagem para realizar investigações científicas que, por sua vez, explica-se pelo processo tardio de cientifização que marcou a história da enfermagem. Vale ressaltar também a influência que a atual política da Universidade exerce nesse contexto, exacerbando as exigências de produtividade, reduzida esta a número de trabalhos publicados, o que tende a gerar pesquisas puramente descritivas (mais rápidas de serem feitas). A terceira característica apontada ocorre não apenas por aquele departamento possuir um maior número de docentes, mas também por haver um maior número deles envolvidos com a iniciação científica. Finalmente, a última característica deve-se ao maior número de bolsas destinadas à Escola de Enfermagem de Ribeirão Preto, comparado ao da Escola de Enfermagem de São Paulo (respectivamente, 57 e 39, no período enfocado).

Tendo em vista o reconhecimento generalizado da importância da pesquisa de Iniciação Científica na formação profissional do aluno, buscamos, neste trabalho, caracterizar essa produção e analisá-la criticamente com vistas a instigar o debate sobre ela e contribuir para a superação de seus pontos vulneráveis.

Podemos afirmar que esta investigação permitiu o desenvolvimento de um trabalho autêntico de equipe e proporcionou os alicerces necessários à formação em pesquisa de seus bolsistas de Iniciação Científica.

\section{SCIENTIFIC INITIATION IN THE NURSING UNDERGRADUATE PROGRAMME OF THE UNIVERSITY OF SÃO PAULO (1993 - 1996): CRITICAL ANALYSIS}

This study search to delineate the profile of the scientific production of the undergraduate nursing programme of the University of São Paulo from 1993 to 1996, identifying its main lines and tendencies. In order to characterize it, the summaries of the proceedings of the Scientific Initiation Symposium of USP were analyzed (SICUSP) and of the Symposum of the University of São Paulo at Ribeirão Preto College of Nursing (SICEERP), through a classification in research types, themes and departments of origin. We observed a prevalence of descriptive research and an expressive number of investigations directed to the study of socio-cultural aspects among other results.

\section{LA INICIACIÓN CIENTÍFICA EN EL PRE-GRADO DE ENFERMERÍA DE LA UNIVERSIDAD DE SÃO PAULO (1993 A 1996): ANÁLISIS CRÍTICO}

Este estudio busca delinear el perfil de la producción científica de pregrado en Enfermería en la Universidad de San Pablo de 1993 a 1996, identificando los trazos principales y sus tendencias. Para esa caracterización fueron analizados los resúmenes de artículos de los Simposios de Iniciación Científica de la USP (SICUSP) y de los Simposios de Iniciación Científica de la Escuela de Enfermería de Ribeirão Preto (SICEERP), a través de una clasificación por tipos de investigación, temáticas y departamentos de origen. Observamos un predominio de investigaciones de tipo descriptivo, y un número expresivo de trabajos volcados para el estudio de aspectos socioculturales dentro de otros resultados.

TÉRMINOS CLAVES: investigación, estudiante de enfermería

\section{REFERÊNCIAS BIBLIOGRÁFICAS}

01. ANGERAMI, E.L.S. Marco teórico das investigações em enfermagem, 1991. p.16 (mimeografado).

02. CHIZZOTTI, A. Pesquisa em ciências humanas e sociais. 2.ed. São Paulo: Cortez, 1995. 164p.
03.CONSELHO NACIONAL DO DESENVOLVIMENTO CIENTÍFICO E TECNOLÓGICO (CNPq). Diretoria de desenvolvimento científico. Manual do usuário. Brasília, abril. 1996.

04. KERLINGER, F.N. Foundations of behavioral research. New York: Holt, Rinehart and Winston, 1964. 521p. 
05. NOGUEIRA, M.J. de C. A pesquisa em enfermagem no Brasil: retrospectiva histórica. Rev. Esc. Enfermagem USP, São Paulo, v.16, p.17-26, 1982.

06. SILVA, G.B.da. Lux et umbrae: o ardil universitário e outros ardis. Ribeirão Preto, 1994. 194p. Tese (Livre Docência) - Escola de Enfermagem de Ribeirão Preto, Universidade de São Paulo.

07. SIMPÓSIO DE INICIAÇÃO CIENTÍFICA DA ESCOLA DE ENFERMAGEM DE RIBEIRÃO PRETO 1, Ribeirão Preto, 1993. Programa e resumo. Ribeirão Preto, 1993.

08. SIMPÓSIO DE INICIAÇÃO CIENTÍFICA DA ESCOLA DE ENFERMAGEM DE RIBEIRÃO PRETO 2, Ribeirão Preto, 1994. Programa e resumo. Ribeirão Preto, 1994.

09. SIMPÓSIO DE INICIAÇÃO CIENTÍFICA DA ESCOLA DE ENFERMAGEM DE RIBEIRÃO PRETO 3, Ribeirão Preto, 1993. Programa e resumo. Ribeirão Preto, 1995.

10. SIMPÓSIO DE INICIAÇÃO CIENTÍFICA DA UNIVERSIDADE DE SÃO PAULO 1., São Paulo, maio 1993. Programa e resumos. São Paulo, 1993.
11. SIMPÓSIO DE INICIAÇÃO CIENTÍFICA, DA UNIVERSIDADE DE SÃO PAULO 2., São Paulo, 1994. Programa e resumos. São Paulo, 1994.

12. SIMPÓSIO DE INICIAÇÃO CIENTÍFICA, DA UNIVERSIDADE DE SÃO PAULO 3., São Paulo, 1995. Programa e resumos. São Paulo, 1995.

13. SIMPÓSIO DE INICIAÇÃO CIENTÍFICA DA UNIVERSIDADE DE SÃO PAULO, 4., São Paulo, 1996. Programa e resumos. São Paulo, 1996.

14. ThiOllent, M.J.M. Crítica metodológica, investigação social e enquete operária. 3.ed. São Paulo: Polis, 1982. 270p.

15. UNIVERSIDADE DE SÃO PAULO. Anuário estatístico. 1996. São Paulo, USP, 1996.

16. UNIVERSIDADE DE SÃO PAULO. Instituto de Química de São Carlos. Comissão de Pesquisa. Boletim Informativo da Comissão de Pesquisa. Agências de Fomento à Pesquisa. São Carlos, 1996. 